\title{
Photoionization of furan from the ground and excited electronic states
}

Cite as: J. Chem. Phys. 144, 084307 (2016); https://doi.org/10.1063/1.4941608

Submitted: 13 November 2015 . Accepted: 27 January 2016 . Published Online: 25 February 2016

Aurora Ponzi, Marin Sapunar, Celestino Angeli (D), Renzo Cimiraglia, Nađa Došlić, and Piero Decleva (D)
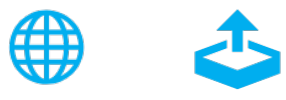

\section{ARTICLES YOU MAY BE INTERESTED IN}

Dynamical photoionization observables of the CS molecule: The role of electron correlation The Journal of Chemical Physics 140, 204304 (2014); https://doi.org/10.1063/1.4876495

Excited state non-adiabatic dynamics of pyrrole: A time-resolved photoelectron spectroscopy and quantum dynamics study

The Journal of Chemical Physics 142, 074302 (2015); https://doi.org/10.1063/1.4907529

Dyson orbitals for ionization from the ground and electronically excited states within equation-of-motion coupled-cluster formalism: Theory, implementation, and examples The Journal of Chemical Physics 127, 234106 (2007); https://doi.org/10.1063/1.2805393

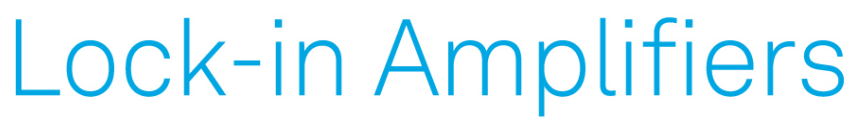
... and more, from DC to $600 \mathrm{MHz}$ Watch 


\title{
Photoionization of furan from the ground and excited electronic states
}

\author{
Aurora Ponzi, ${ }^{1}$ Marin Sapunar, ${ }^{1}$ Celestino Angeli, ${ }^{2}$ Renzo Cimiraglia, ${ }^{2}$ Nađa Došlić, ${ }^{1}$ and \\ Piero Decleva ${ }^{3}$ \\ ${ }^{1}$ Department of Physical Chemistry, R. Bošković Institute, Bijenička 54, 10000 Zagreb, Croatia \\ ${ }^{2}$ Dipartimento di Scienze Chimiche e Farmaceutiche, Università di Ferrara, via Fossato di Mortara 17, \\ 44121 Ferrara, Italy \\ ${ }^{3}$ Dipartimento di Scienze Chimiche e Farmaceutiche, Università degli Studi di Trieste, via L. Giorgieri 1, \\ 34127 Trieste, Italy
}

(Received 13 November 2015; accepted 27 January 2016; published online 25 February 2016)

\begin{abstract}
Here we present a comparative computational study of the photoionization of furan from the ground and the two lowest-lying excited electronic states. The study aims to assess the quality of the computational methods currently employed for treating bound and continuum states in photoionization. For the ionization from the ground electronic state, we show that the Dyson orbital approach combined with an accurate solution of the continuum one particle wave functions in a multicenter B-spline basis, at the density functional theory (DFT) level, provides cross sections and asymmetry parameters in excellent agreement with experimental data. On the contrary, when the Dyson orbitals approach is combined with the Coulomb and orthogonalized Coulomb treatments of the continuum, the results are qualitatively different. In excited electronic states, three electronic structure methods, TDDFT, ADC(2), and CASSCF, have been used for the computation of the Dyson orbitals, while the continuum was treated at the B-spline/DFT level. We show that photoionization observables are sensitive probes of the nature of the excited states as well as of the quality of excited state wave functions. This paves the way for applications in more complex situations such as time resolved photoionization spectroscopy. @ 2016 AIP Publishing LLC. [http://dx.doi.org/10.1063/1.4941608]
\end{abstract}

\section{INTRODUCTION}

Coupling of electron and nuclear motion is fundamental to our understanding of many photoinduced reactions in chemistry and biochemistry. In this context, on-the-fly nonadiabatic (NA) dynamics has been increasingly used for elucidating the electronic and structural changes that molecular systems undergo after photoexcitation. ${ }^{1-4}$ Although the detailed physical insight provided by NA dynamics simulations has played a key role in discovering new photochemical mechanisms, from the quantitative point of view, simulations based on various electronic structure methods and dynamics algorithms have frequently provided different and even contrasting results on the mechanisms and time scale of nonradiative relaxation of prototype photochemical molecules. ${ }^{5-7} \mathrm{~A}$ notable example is the much studied excited state deactivation of $9 \mathrm{H}$-adenine where a recent analysis has revealed that the large variation in the results was primarily due to erroneous potential energy surface topographies and, thus, to the electronic structure methods implemented in dynamics simulations. ${ }^{6}$ Also, the comparison between measured and computed lifetimes is complicated by the difficulties to reproduce specific experimental conditions such as excitations accessing portions of the configurational space away from the origin of the targeted transition or the Franck-Condon region. ${ }^{8}$ Altogether, in order to establish a direct link between dynamics simulations and experiments, observables pertinent to the specific probe processes have to be computed.
In this respect, time-resolved photoelectron spectroscopy (TRPES) is a particularly suitable detection technique, ${ }^{9-11}$ which has been extensively employed in studies of ultrafast dynamics of small and medium size molecules. ${ }^{12-19}$ In TRPES, the system is initially excited to a bright electronic state. After a variable delay time, a high-energy probe ionizes the molecule from its current electronic state by absorption of a single photon. The scattered photoelectrons, measured as a function of time, carry the signature of the orbital structure of the molecule allowing the determination of the lifetimes. ${ }^{20}$ Moreover, by encoding information about the molecular electronic structure, cross sections and angular distributions can be used for testing the quality of molecular wave functions employed in the dynamics simulations. ${ }^{21}$ However, extracting molecular configurations from experimental data requires a high-level theoretical analysis which calls not only for an accurate description of the initial, $\Psi^{N}$, and final, $\Psi^{N-1}$, bound states, but also for a careful treatment of the molecular continuum. ${ }^{22}$

Recently, several theoretical investigations have addressed simulation of TRPES. ${ }^{16,19,23-25}$ Due to the difficulty of describing the ionization from excited states, which are often of multiconfigurational character, a single channel approach has been invariably employed. In this case, photoionization transition matrix elements can be well described (neglecting the conjugate process) as a dipole transition from an initial orbital, the so called Dyson orbital relative to the initial excited and final ionic bound states, to final continuum orbitals. The Dyson orbital can be computed relatively easily even from 
highly correlated wave functions. ${ }^{21,24-28}$ Evaluation of the continuum is more difficult, and computationally expensive. To simplify this step, studies have employed either Stieltjes imaging, ${ }^{29}$ which cannot provide angular distributions, or just plane or Coulomb waves, whose accuracy is however uncertain. ${ }^{23,24,26,28}$ Although used in the past, at the beginning of photoionization studies, these approximations often turned out quite poor, and have been practically abandoned in normal photoionization studies, although enjoying a renewed popularity in the more complex context of TRPES.

In this work, we focus on the photoionization of furan, a prototypical photochemical system, both from the ground and lowest-lying excited electronic states. ${ }^{16,30,31}$ We will use the Dyson orbitals approach together with an accurate description of the molecular continuum obtained using a multicentric basis of B-spline functions to evaluate cross sections and asymmetry parameters. ${ }^{22,32}$ Apart from the ground state minimum energy structure, we considered a bent structure that is typically encountered along the relaxation pathway of furan after optical excitation to the bright $\mathrm{B}_{2}\left(\pi \pi^{*}\right)$ state. The multicentric B-spline approach has proven to be a very accurate way to describe single-electron ionization problems. Here it is used for the first time in the context of molecular photoionization from excited electronic states in order to provide benchmark results against which more approximate, but also more efficient methods, could be compared. Further, our calculations aim to test the sensitivity of photoionization observables on the description of the molecular electronic structure. Thus, the initial and final bound states have been computed using electronic structure methods typically employed in NA dynamics simulations: complete active space self-consistent field (CASSCF), timedependent density functional theory (TDDFT), and the algebraic diagrammatic construction to the second order (ADC(2)) method. ${ }^{33,34}$ The quality of the description of the continuum is gauged by the comparison with experimental results, both branching ratios and asymmetry parameters, for ground state ionization, and then used as a benchmark to compare with Coulomb and orthogonalized Coulomb waves currently employed in such studies.

\section{THEORETICAL APPROACH}

\section{A. Overview}

This work focuses on the calculation of the dynamical observables from the ground and lowest-lying excited states of furan. For the ground state, the different states involved in the photoionization process have been described by the following two procedures which differ in the treatment of the bound states. In the first procedure, the bound states were calculated by density functional theory (DFT) with a multi-center basis of B-splines functions (Sec. II C). In the second, they were computed using CASSCF calculations followed by the calculation of the relative Dyson orbitals. In both procedures, the continuum functions were obtained as a continuum solution of the Kohn-Sham (KS) Hamiltonian built from the DFT ground state density. In addition, continuum TDDFT calculations ${ }^{35}$ have been performed to check the importance of the electronic response at the DFT level.
Finally, we have considered simpler treatments for the continuum states, like the use of Coulomb (CW) or Orthogonalized Coulomb waves (OCW) widely employed in the more demanding TRPES calculations, to assess the impact of the different descriptions on the photoionization observables. These have been computed with the same Bspline code, by employing a single center expansion of the initial DFT orbitals, and a hydrogenic continuum for the final state. Additional OCW calculations have been performed by simply shifting the origin of the reference system to annihilate the dipole expectation value of the initial orbital, as suggested in Ref. 24.

In the second part, we have considered the photoionization of furan from excited electronic states at two geometries: the planar equilibrium geometry and a puckered one. Here, the initial and final bound states were computed at the CASSCF, ADC(2), and TDDFT levels. As before, the continuum states were treated at the B-spline/DFT level.

\section{B. Dyson orbitals computation}

The Dyson orbital approach for the computation of photoionization observables has been recently described in a number of publications. ${ }^{21,26,28}$ Thus, here we present only the main steps. The computation of differential cross sections and asymmetry parameters for the ejection of an electron from an initial $N$-electron state, $\Psi_{I}^{N}$, to a continuum state, $\Psi_{E F}^{N}$, by a photon of energy $E$ requires the evaluation of dipole transition moments

$$
D_{I F j}=\hat{n}\left\langle\Psi_{E F j}^{N}|\hat{D}| \Psi_{I}^{N}\right\rangle,
$$

where $\hat{n}$ is the direction of the laser polarization and $\hat{D}$ is the dipole operator which is a sum of single-particle operators $d_{k}, k=1, N$. In the single channel approximation, the correlation between the ejected photoelectron and the $N-1$ electrons of the cation is neglected and the final state can be written as an antisymmetrized product of the form

$$
\Psi_{E F j}^{N}=A \Psi_{F}^{N-1} \phi_{\epsilon j},
$$

where $\Psi_{F}^{N-1}$ is the bound cationic wave function and $\phi_{\epsilon j}$ is the continuum wave function of the photoelectron with kinetic energy $\epsilon$. The index $j$ counts the independent continuum channels (angular momenta). It is now convenient to define $\hat{a}_{k}$ as the annihilation operator of the molecular orbital $\phi_{k}$ and the so-called direct

$$
\chi_{F k}=\left\langle\Psi_{F}^{N-1}\left|\hat{a}_{k}\right| \Psi_{I}^{N}\right\rangle
$$

and conjugated

$$
\eta_{F k}=\left\langle\Psi_{F}^{N-1}\left|\hat{D} \hat{a}_{k}\right| \Psi_{I}^{N}\right\rangle
$$

amplitudes. In terms of amplitudes $\chi_{F k}$ and $\eta_{F k}$ and for a final state of form (2), the dipole matrix elements reduce to single particle matrix elements of the form

$$
\left\langle\Psi_{F}^{N-1} \phi_{\epsilon j}|\hat{D}| \Psi_{I}^{N}\right\rangle=\sum_{k}\left\langle\phi_{\epsilon j}|d| \phi_{k}\right\rangle \chi_{F k}+\sum_{k}\left\langle\phi_{\epsilon j} \mid \phi_{k}\right\rangle \eta_{F k} .
$$


By retaining only the direct term which includes the dipole transition to the continuum wave function, one obtains

$$
\left\langle\Psi_{F}^{N-1} \phi_{\epsilon j}|\hat{D}| \Psi_{I}^{N}\right\rangle=\left\langle\phi_{\epsilon j}|d| \chi_{F}\right\rangle,
$$

where $\chi_{F}=\sum_{k} \chi_{F k} \phi_{k}$ defines the single particle Dyson orbital which is basically an effective orbital for the hole created in $\Psi_{F}^{N-1}$. The norm of the Dyson orbital, $\sum_{k}\left|\chi_{F k}\right|^{2}$, defines the spectral strength, i.e., the probability to observe the $\Psi_{F}^{N-1}$ state in the sudden approximation.

Let us now consider the excited states of the neutral molecule as initial states of the ionization process. The computation of the Dyson orbitals in the framework of linear-response TDDFT relies on the concept of auxiliary wave function. ${ }^{36,37}$ The auxiliary wave function of the jth excited state is given as a linear combination of singly excited Slater determinants constructed from the occupied (i) and unoccupied (a) KS orbitals

$$
\Psi_{I}^{j, N}=\sum_{i, a} c_{i, a}^{j} a_{a}^{\dagger} a_{i}\left|\Phi_{0}^{N}\right\rangle
$$

where $\left|\Phi_{0}^{N}\right\rangle$ is the reference KS determinant. The excitation energies $\omega^{j}$ and eigenvectors $c^{j}$ are obtained by solving the linear-response TDDFT equations in the Casida formalism. At the ADC(2) level, the Hartree-Fock (HF) determinant was used as the reference wave function. Note that Eq. (7) includes only single excitations as in the Turbomole implementation of ADC(2) doubles amplitudes are not available. ${ }^{34}$ The cationic states were described by single, optimized (relaxed) KS or HF determinants. Finally, to compute the overlap needed for the construction of the Dyson orbitals, the wave function of the N-electron system was expanded in minors. ${ }^{24}$ For the calculation of the Dyson orbitals at the CASSCF level, the overlap between the CASSCF wave functions separately optimized for the neutral molecule and for the ion has been evaluated as described previously. ${ }^{21}$

To facilitate the evaluation of the one-particle dipole matrix elements, the TDDFT, ADC(2), and CASSCF Dyson orbitals have been projected onto the B-spline basis. Consequently, the calculation of Dyson orbitals is decoupled from that of continuum dipole matrix elements and can be easily treated using different electronic structure methods.

\section{B-spline DFT approach}

The computational procedure based on the solution of the scattering problem at the static-exchange DFT level provides a quantitative description of the dynamical photoionization observables and generally produces results in excellent agreement with experiment. ${ }^{38,39}$ As the method has been discussed in detail in previous reports, we outline only the essential steps. ${ }^{22,32}$

At the static-exchange DFT level, both bound and continuum orbitals are obtained as eigenfunctions of a single KS Hamiltonian, $h_{K S}$, defined by the ground-state electron density $\rho$ as

$$
h_{K S}=-\frac{1}{2} \Delta+V_{N}+V_{C}+V_{X C}
$$

In this expression, $V_{N}$ is the nuclear attraction potential, $V_{C}(\rho)$ is the Coulomb potential and $V_{X C}(\rho)$ is the exchangecorrelation potential. The SCF initial electronic density of the ground state has been obtained from the ADF program. ${ }^{40,41}$

In the present method, the wave function is expanded on a basis set of a product of radial B-spline functions and real spherical harmonics adapted to the symmetry. The primitive basis functions can then be expressed as

$$
\chi_{i l m}=\frac{1}{r} B_{i}(r) Y_{l m}(\theta, \phi) .
$$

The radial and angular parts of the basis set are expanded over several suitably chosen centers. These correspond to a common origin (long-range one-center expansion), with large angular momenta, $\left\{\chi_{n l m}^{0}\left(\mathbf{r}_{0}\right)\right\}$, and to a set of short-range functions which are located at the nuclei, $\left\{\chi_{n l m}^{p}\left(\mathbf{r}_{p}\right)\right\}$. The short-range expansions properly describe the Coulomb cusps of the wave function at the nuclei and dramatically improve the convergence of the one-center expansion. This choice allows one to correctly describe the bound states as well as the long range behavior of the continuum wave functions.

Both the bound states

$$
h_{K S} \phi_{n \lambda \mu}=E_{n \lambda} \phi_{n \lambda \mu}
$$

and the continuum states

$$
h_{K S} \phi_{E \lambda \mu}=E \phi_{E \lambda \mu}
$$

are obtained by converting the differential equations into the corresponding matrix equations,

$$
\begin{gathered}
\phi_{i}=\sum_{k} \chi_{k} c_{k i}, \quad H_{k l}=\left\langle\chi_{k}\left|h_{K S}\right| \chi_{l}\right\rangle, \\
S_{k l}=\left\langle\chi_{k} \mid \chi_{l}\right\rangle, \quad H c=E S c .
\end{gathered}
$$

By means of a generalized diagonalization of the Hamiltonian, one can solve the KS equations for the bound states. The resulting KS orbitals are expanded in the full basis set. The full set of independent continuum states can be obtained by using the Galerkin approach, ${ }^{42-44}$ and is then transformed to K-matrix boundary conditions. Finally, the photoionization observables (cross sections and asymmetry parameters) are calculated from the transition dipole moments according to standard formulas. ${ }^{45}$

\section{COMPUTATIONAL DETAILS}

DFT calculations have been carried out using the ADF code. ${ }^{40,41}$ We have employed the exchange-correlation potential LB94 ${ }^{46}$ and, for each atom, the DZP STO basis set, taken from the optimized database included in the ADF package. Once the ground-state electron density is obtained, both occupied and continuum orbitals were calculated in the B-spline LCAO basis. A long-range one-center expansion with asymptotic angular expansion up to $\mathrm{L}=15$, an interval up to $\mathbf{R}_{\max }=25$ a.u., and step size of 0.2 a.u. has been chosen. With the origin of the coordinate system at the oxygen atom, the off-center functions were added around the carbon and hydrogen atoms, with $\mathrm{L}_{\max }=2$ and $\mathrm{L}_{\max }=1$, respectively. In the planar case, the radial expansions around the atoms were $\mathrm{R}_{\max }=1.0$ a.u. for the carbon atoms and $\mathrm{R}_{\max }=0.5$ a.u. for the 
hydrogen atoms. For the bent geometry, values of $\mathrm{R}_{\max }=0.8$, 0.6 , and 0.5 a.u. were used for $\mathrm{O}, \mathrm{C}$, and $\mathrm{H}$-atoms, respectively. These choices permit to obtain an accurate solution of both the discrete and continuum orbitals. For the ground state ionization, we have also performed a set of calculation based on TDDFT. For these calculations, a previously developed non-iterative algorithm has been used. ${ }^{35}$

For the calculations of vertical excitation energies and ionization energies in the $\mathrm{C}_{2 v}$ symmetry, we have used the standard Dunning correlation-consistent basis set augmented with polarization and diffuse functions (aug-cc-pVDZ) for $\mathrm{O}$, $\mathrm{C}$, and $\mathrm{H}$ atoms. ${ }^{47}$ The corresponding CASSCF orbitals were obtained by treating six valence electrons as active electrons which are distributed among nine orbitals: one $a_{1}$, four $b_{1}$, one $b_{2}$, and three $a_{2}$, i.e., 3 occupied $\left(1 b_{1}-2 b_{1}, 1 a_{2}\right)$ and 6 virtual $\left(1 a_{1}, 3 b_{1}-4 b_{1}, 7 b_{2}, 2 a_{2}-3 a_{2}\right)$. The same active space has been selected for the bent geometry. Both the excitation and ionization energies have been corrected by including the residual dynamical correlation contribution through the NEVPT2 multireference perturbation theory, at the Partially Contracted (PC(2)) level. ${ }^{48,49}$

HF, CASSCF, and NEVPT2 calculations have been performed with the MOLPRO package ${ }^{33}$ and with special modules developed in our laboratories. TDDFT and ADC(2) calculations have been performed with Turbomole. ${ }^{34}$

\section{RESULTS AND DISCUSSION}

\section{A. Photoionization from the ground electronic state}

According to previous calculations, ${ }^{30}$ the valence shell molecular orbital sequence of furan in its ground state $\left(\mathrm{C}_{2 v}\right.$ symmetry) can be written as

$$
\begin{aligned}
& \left(4 a_{1}\right)^{2}\left(5 a_{1}\right)^{2}\left(3 b_{2}\right)^{2}\left(4 b_{2}\right)^{2}\left(6 a_{1}\right)^{2}\left(7 a_{1}\right)^{2}\left(1 b_{1}\right)^{2} \\
& \quad \times\left(5 b_{2}\right)^{2}\left(6 b_{2}\right)^{2}\left(8 a_{1}\right)^{2}\left(9 a_{1}\right)^{2}\left(2 b_{1}\right)^{2}\left(1 a_{2}\right)^{2} .
\end{aligned}
$$

Here, we consider the outer valence shell composed of five orbitals. The $1 a_{2}$ and $2 b_{1} \pi$ orbitals constitute the HOMO and HOMO-1, respectively. The former derives from the $\pi$ electrons on the carbon atoms $\mathrm{C}_{\beta}$ and $\mathrm{C}_{\alpha}$ (see Fig. 1 and Table I), whereas the $\mathrm{C}_{\alpha}$ and oxygen lone-pair electrons form the main contribution to the HOMO-1 orbital.

These orbitals give rise to two well resolved photoelectron bands in the spectrum of furan, while a more complex structure stems from ionization from the next outer valence orbitals. ${ }^{30}$ These $\left(6 \mathrm{~b}_{2}, 8 \mathrm{a}_{1}\right.$, and $\left.9 \mathrm{a}_{1}\right)$, having $\sigma$-character, also contain contribution from the $\mathrm{H} 1 \mathrm{~s}$ orbitals, as reported in Table I.

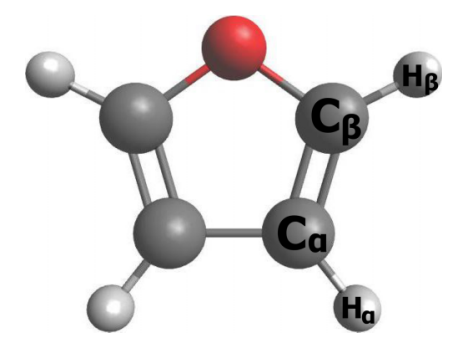

FIG. 1. Furan molecule.
TABLE I. Orbital composition of the five outermost orbitals of furan (equilibrium geometry).

\begin{tabular}{lccc}
\hline \hline MO & Percentage & AO & Atom \\
\hline $1 \mathrm{a}_{2}$ (HOMO) & 68.39 & $2 \mathrm{p}_{x}$ & $\mathrm{C}_{\beta}$ \\
& 25.27 & $2 \mathrm{p}_{x}$ & $\mathrm{C}_{\alpha}$ \\
\hline $2 \mathrm{~b}_{1}($ HOMO-1) & 70.14 & $2 \mathrm{p}_{x}$ & $\mathrm{C}_{\alpha}$ \\
& 22.04 & $2 \mathrm{p}_{x}$ & $\mathrm{O}$ \\
\hline $9 \mathrm{a}_{1}$ & 25.24 & $2 \mathrm{p}_{z}$ & $\mathrm{O}$ \\
& 19.80 & $2 \mathrm{p}_{y}$ & $\mathrm{C}_{\alpha}$ \\
& 16.95 & $2 \mathrm{p}_{z}$ & $\mathrm{C}_{\beta}$ \\
& 14.23 & $2 \mathrm{p}_{z}$ & $\mathrm{C}_{\alpha}$ \\
& 10.30 & $1 \mathrm{~s}$ & $\mathrm{H}_{\beta}$ \\
\hline $8 \mathrm{a}_{1}$ & 36.78 & $2 \mathrm{p}_{y}$ & $\mathrm{C}_{\alpha}$ \\
& 27.54 & $2 \mathrm{p}_{z}$ & $\mathrm{O}$ \\
& 18.16 & $1 \mathrm{~s}$ & $\mathrm{H}_{\alpha}$ \\
\hline $6 \mathrm{~b}_{2}$ & 27.50 & $2 \mathrm{p}_{z}$ & $\mathrm{C}_{\alpha}$ \\
& 24.51 & $1 \mathrm{~s}$ & $\mathrm{H}_{\alpha}$ \\
& 15.52 & $2 \mathrm{p}_{z}$ & $\mathrm{C}_{\beta}$ \\
& 10.64 & $2 \mathrm{p}_{y}$ & $\mathrm{C}_{\beta}$ \\
\hline \hline
\end{tabular}

The ionization energies for the first two ionic states of furan, ${ }^{2} \mathrm{~A}_{2}$ and ${ }^{2} \mathrm{~B}_{1}$, have been computed with the PC-NEVPT2 method. ${ }^{48,49}$ They were obtained as the difference between the absolute energy of each cation and the ground state energy of the neutral molecule. As seen from Table II, CASSCF ionization energies deviate from the experimental values by about 0.6 and $0.9 \mathrm{eV}$ for the first and the second ionizations, respectively. The ionization energies calculated with the NEVPT2 approach are in excellent agreement with the experimental values as the method takes into account the ground state dynamical correlation not accounted for at the CASSCF level.

\section{B. Branching ratios and asymmetry parameters}

The experimental and theoretical photoelectron branching ratios corresponding to the five outermost orbitals of furan are plotted in Figure 2. These observables represent the ratio between a specific cross section and the sum of all considered cross sections, determined as a function of photon energy. The theoretical values have been calculated at the DFT and TDDFT levels and, in both cases, the continuum states have been computed with the B-spline/DFT approach. The branching ratios for the HOMO and HOMO-1 orbitals present small variations as a function of energy and do not deviate significantly from the value of 0.1 . The curves related to the DFT and TDDFT calculations fit perfectly with the experimental ones. The branching ratio profiles for the $\sigma$ -

TABLE II. Ionization energies of furan in $\mathrm{eV}$.

\begin{tabular}{lccr}
\hline \hline State & CAS-[6,9] & NEVPT2 & Expt. $^{\mathrm{a}}$ \\
\hline${ }^{2} \mathrm{~A}_{2}$ & 8.14 & 8.86 & 8.78 \\
${ }^{2} \mathrm{~B}_{1}$ & 9.43 & 10.42 & 10.38 \\
\hline \hline
\end{tabular}

${ }^{\mathrm{a}}$ Reference 50 


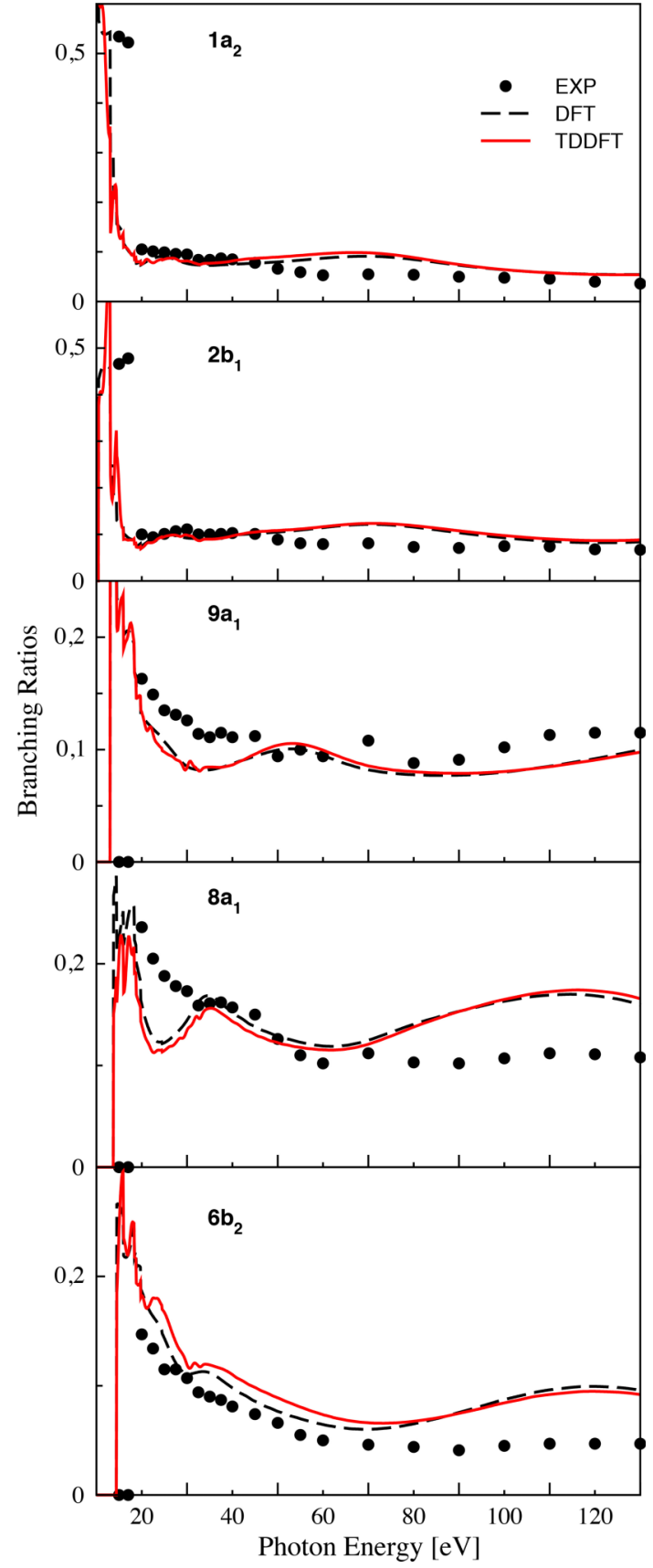

FIG. 2. The branching ratios of furan for the $1 \mathrm{a}_{2}$ (HOMO), $2 \mathrm{~b}_{1}$ (HOMO-1), $9 \mathrm{a}_{1}, 8 \mathrm{a}_{1}$, and $6 \mathrm{~b}_{2}$ orbitals.

character orbitals $\left(6 \mathrm{~b}_{2}, 8 \mathrm{a}_{1}\right.$, and $\left.9 \mathrm{a}_{1}\right)$ exhibit some oscillating behavior which differs from the one of the $\pi$-orbitals. Their dependence on energy value is much more pronounced: the BR patterns start from a value of $\sim 0.2-0.3$ and then decrease to reach the mean value of $\sim 0.1$. For these three orbitals, some deviations of the calculated values compared to those measured can be observed at high energy and, in the case of $9 a_{1}$ and $8 a_{1}$ orbitals, there is also a deviation at around 20-40 eV. Due to the only partly resolved nature of the composite band and the relatively sparse energy points, it is also possible that some details are missing in the experimental results.

Next, we have performed two sets of calculations for the asymmetry parameters $(\beta)$ of the five outermost orbitals. The results, compared with the experimental data, are presented in Figure 3. Again, the dependence of a particular asymmetry parameter on the energy reflects the $\sigma$ or $\pi$ character of the orbital from which the ionization occurs. ${ }^{51}$ Asymmetry parameters for HOMO and HOMO-1 orbitals rise rapidly with increasing energy and reach a mean value of $1.8 \beta$ units at around $40 \mathrm{eV}$. On the contrary, the asymmetry parameters associated with the $\sigma$-orbitals rise more slowly with increasing energy. Furthermore, we can observe differences in magnitude and shape in their profiles starting from the threshold region up to $70 \mathrm{eV}$. This trend confirms previous observations showing that, for unsaturated organic molecules, the asymmetry parameters for $\pi$-orbitals increase more rapidly as a function of energy compared to the behavior of $\sigma$-orbitals. ${ }^{51}$ The shape

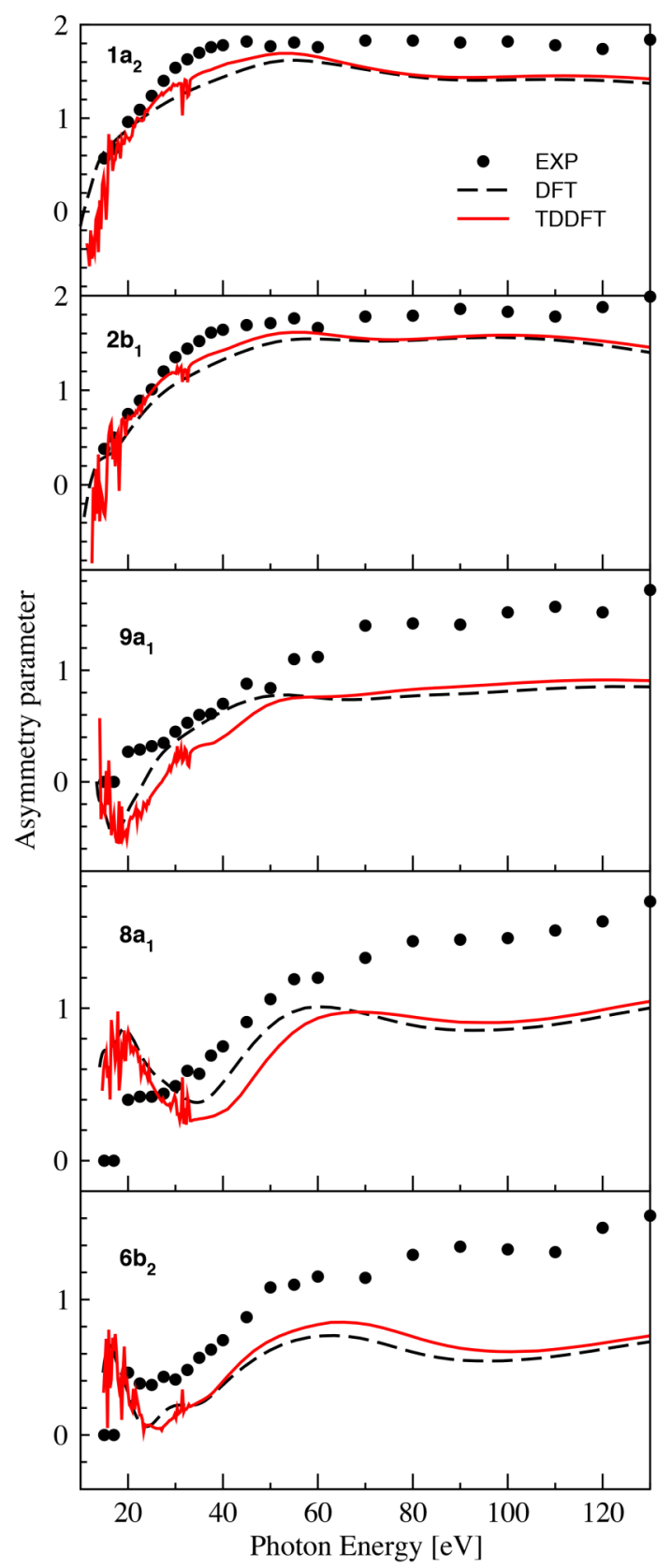

FIG. 3. The asymmetry parameters of furan for the $1 a_{2}$ (HOMO), $2 b_{1}$ (HOMO-1), $9 \mathrm{a}_{1}, 8 \mathrm{a}_{1}$, and $6 \mathrm{~b}_{2}$ orbitals. 
and the absolute values of the experimental points are matched quite well by the theoretical curves especially for the HOMO and HOMO-1 orbitals. Both calculated sets are basically superimposed for all the ionizations considered even if, in the range of photon energy between 40 and $60 \mathrm{eV}$, the asymmetry parameters calculated with the DFT method for the $9 \mathrm{a}_{1}$ and $8 \mathrm{a}_{1}$ orbitals are slightly higher than those obtained using TDDFT. Deviations from the experimental data can be observed for both DFT and TDDFT calculations for the $\sigma$-orbitals, in particular at high energy. However, the overall comparison between theoretical and experimental results shows that our methods give a pretty good description, especially below $60 \mathrm{eV}$ photon energy, of the energy dependence of the photoionization observables and that fingerprints of the nature of the molecular orbitals can be retrieved from the spectral behavior of both the branching ratios and the asymmetry parameters.

\section{Influence of the description of bound and continuum states on the photoionization observables}

Here, we investigate how the treatment of bound and continuum states impacts the cross sections and the asymmetry parameters. The photoionization of furan from the ground state to the first, ${ }^{2} \mathrm{~A}_{2}\left(\mathrm{D}_{0}\right)$, and second, ${ }^{2} \mathrm{~B}_{1}\left(\mathrm{D}_{1}\right)$, ionic states will be considered. Three computational methods, HF, DFT, and Dyson orbitals based on CAS-[6,9], have been used for the description of the bound states in order to study the signature of electron correlation. All three methods predict similar cross section profiles with a relatively smooth decay with energy increase. Cross sections for the ionization from the HOMO and HOMO-1 are shown in the left panels of Figure 4. In both

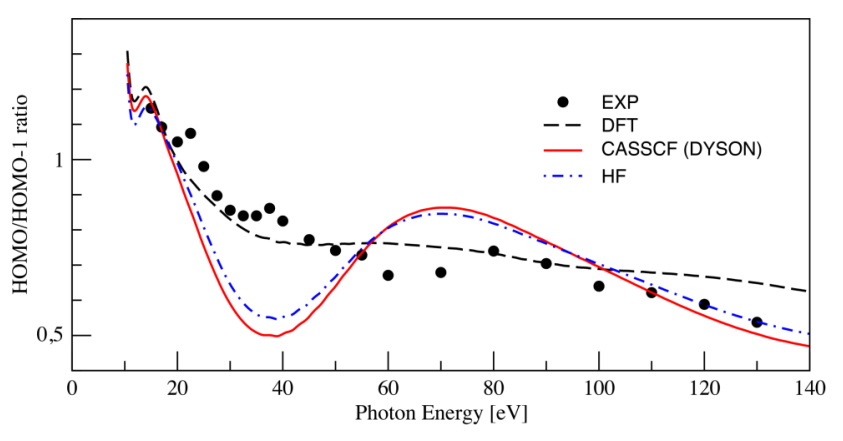

FIG. 5. Experimental and theoretical ratios of the photoionization partial cross sections for the ionization from the HOMO and HOMO-1 orbitals of furan.

cases, values computed with the CASSCF Dyson orbitals are found to be slightly lower in the threshold region. The differences among HF, DFT, and CASSCF Dyson values can be more easily seen when the HOMO/HOMO-1 ratio of the photoionization cross sections is compared to the experimental data as shown in Figure 5. It is evident that, even though the oscillations in the ratio of the cross sections have not been captured by the calculations, the overall experimental profile is reproduced much better by DFT. The oscillation is instead reproduced in the $a b$ initio results, although overestimated and shifted towards threshold. The asymmetry parameters (Figure 4, right panels) of the different methods are in very good agreement. We notice a small discrepancy between the DFT and the HF and CASSCF results for the HOMO orbital in the $10-50 \mathrm{eV}$ energy range. This can be related to the choice of basis set as we have seen from preliminary tests that a large basis set is important to reach convergence even at the
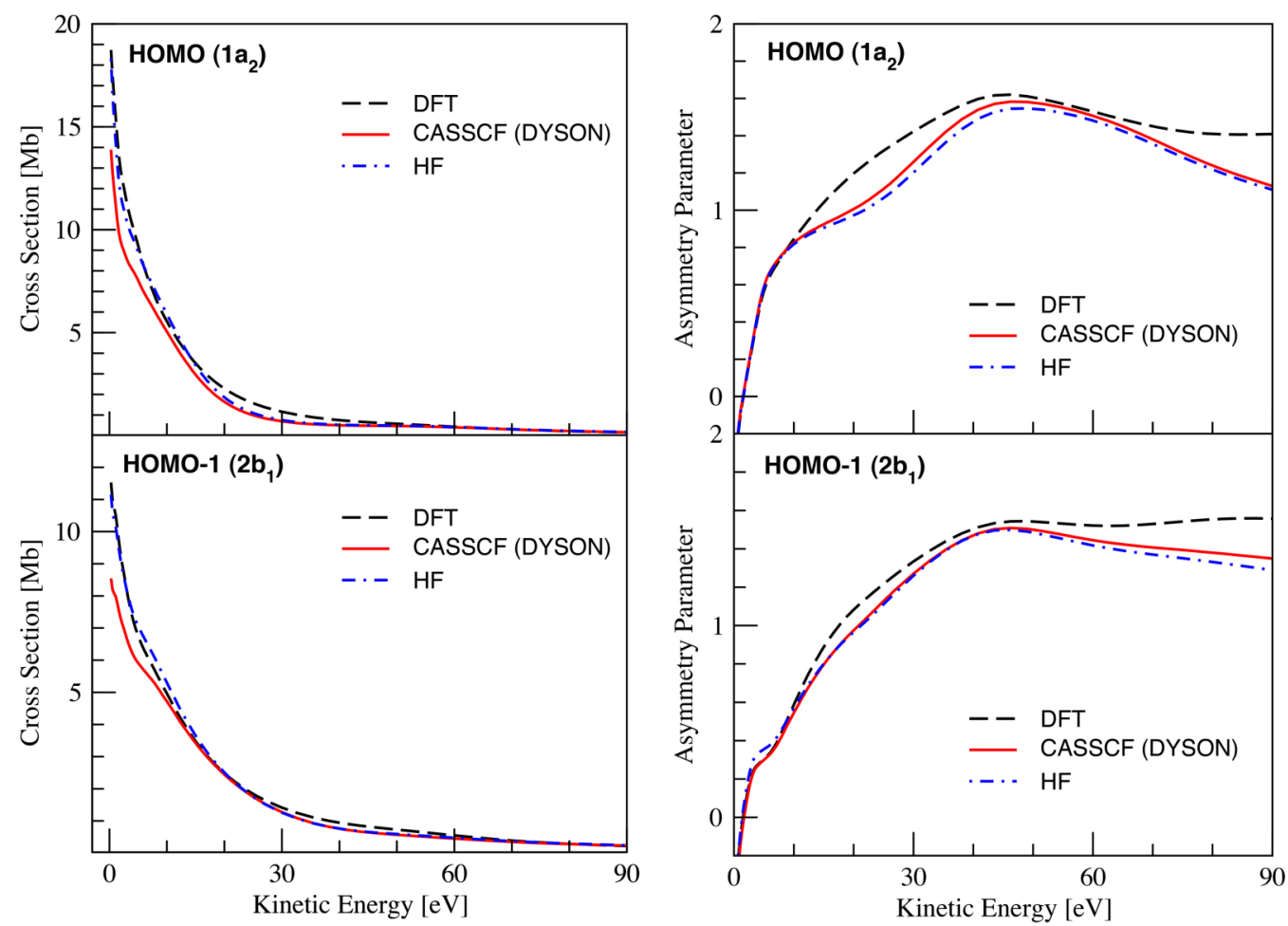

FIG. 4. The theoretical photoionization partial cross sections and photoelectron asymmetry parameters of the HOMO (upper panels) and HOMO-1 (lower panels) orbitals of furan. 
HF level. Regardless, it appears that all three methods used for the description of the bound states perform reasonably well and have a rather modest impact on the description of photoionization observables.

Next, we investigate the effect of the electronic continuum treatment on the photoionization observables. Specifically, we keep the same Dyson orbital approach (using CASSCF) and compare the results obtained using the multi-center B-spline/DFT approach (Figure 4) to the results from the more approximate $\mathrm{CW}$ and $\mathrm{OCW}$ representations of the continuum states. These approximations have been employed in recent TRPES computations. ${ }^{24}$ From Figure 6, it is evident that serious discrepancies exist both in the cross sections and in the asymmetry parameters. A much slower decrease of the cross section values with energy increase is found. Although the orthogonalization of continuum states with respect to the Dyson orbital slightly reduces the discrepancy, the high energy behavior is not reproduced. The effect is even more pronounced in the asymmetry parameters where the rapid rise of the $\beta$ parameter characteristic for $\pi$-orbitals of heterocyclic molecules has not been captured. On the contrary, the asymmetry parameters computed with both Coulomb and orthogonalized Coulomb states resemble more the profile of the $\sigma$ orbitals of furan. Owing to these qualitative differences, the simulations of furan photoionization from excited electronic states have been performed using only the B-spline description of the electronic continuum.

\section{Photoionization from excited electronic states}

Vertical excitation energies of furan at the equilibrium geometry have been evaluated using TDDFT, $\mathrm{ADC}(2)$, and
TABLE III. Excitation energies of furan in eV.

\begin{tabular}{lccccc}
\hline \hline State & CAS-[6,9] & NEVPT2 & TDDFT & ADC(2) & Expt. $^{\text {a }}$ \\
\hline${ }^{1} \mathrm{~A}_{2}$ & 5.71 & 5.98 & 5.51 & 5.91 & 6.04 \\
${ }^{1} \mathrm{~B}_{2}$ & 6.91 & 6.63 & 5.98 & 6.44 & 6.49 \\
\hline
\end{tabular}

${ }^{\mathrm{a}}$ Reference 53

TABLE IV. Pole strength values for the planar and bent geometries of furan.

\begin{tabular}{lccc}
\hline & CASSCF & TDDFT & ADC(2) \\
\hline State & & Planar geometry & \\
S1-D0 & 0.49 & 0.48 & 0.46 \\
S2(S3)-D0 & 0.49 & 0.47 & 0.45 \\
\hline State & & Bent geometry & \\
S1-D0 & 0.35 & 0.45 & 0.32 \\
S2(S3)-D0 & 0.45 & 0.31 & 0.31 \\
\hline \hline
\end{tabular}

CASSCF/NEVPT2 methods. ${ }^{52}$ The results are compiled in Table III. NEVPT2 and ADC(2) provide results of comparable quality and in good agreement with the experimental data, whereas CASSCF and TDDFT deviate from these values by about 0.4 and $0.5 \mathrm{eV}$, respectively. Specifically, at the CASSCF level the first excited state is underestimated by $0.3 \mathrm{eV}$, while the second is overestimated by $0.4 \mathrm{eV}$. In both cases, TDDFT provides values lower than the experimental ones.

At the planar equilibrium geometry, the two lowest-lying excited electronic states of furan are the ${ }^{1} \mathrm{~A}_{2}(\pi 3 s)$ state with Rydberg character $\left(\mathrm{S}_{1}\right)$, and the ${ }^{1} \mathrm{~B}_{2}\left(\pi \pi^{*}\right)$ state $\left(\mathrm{S}_{2}\right)$. As shown by the oscillator strengths, $S_{1}$ is a dark state, while $S_{2}$ is a bright state. According to TRPES measurements, internal
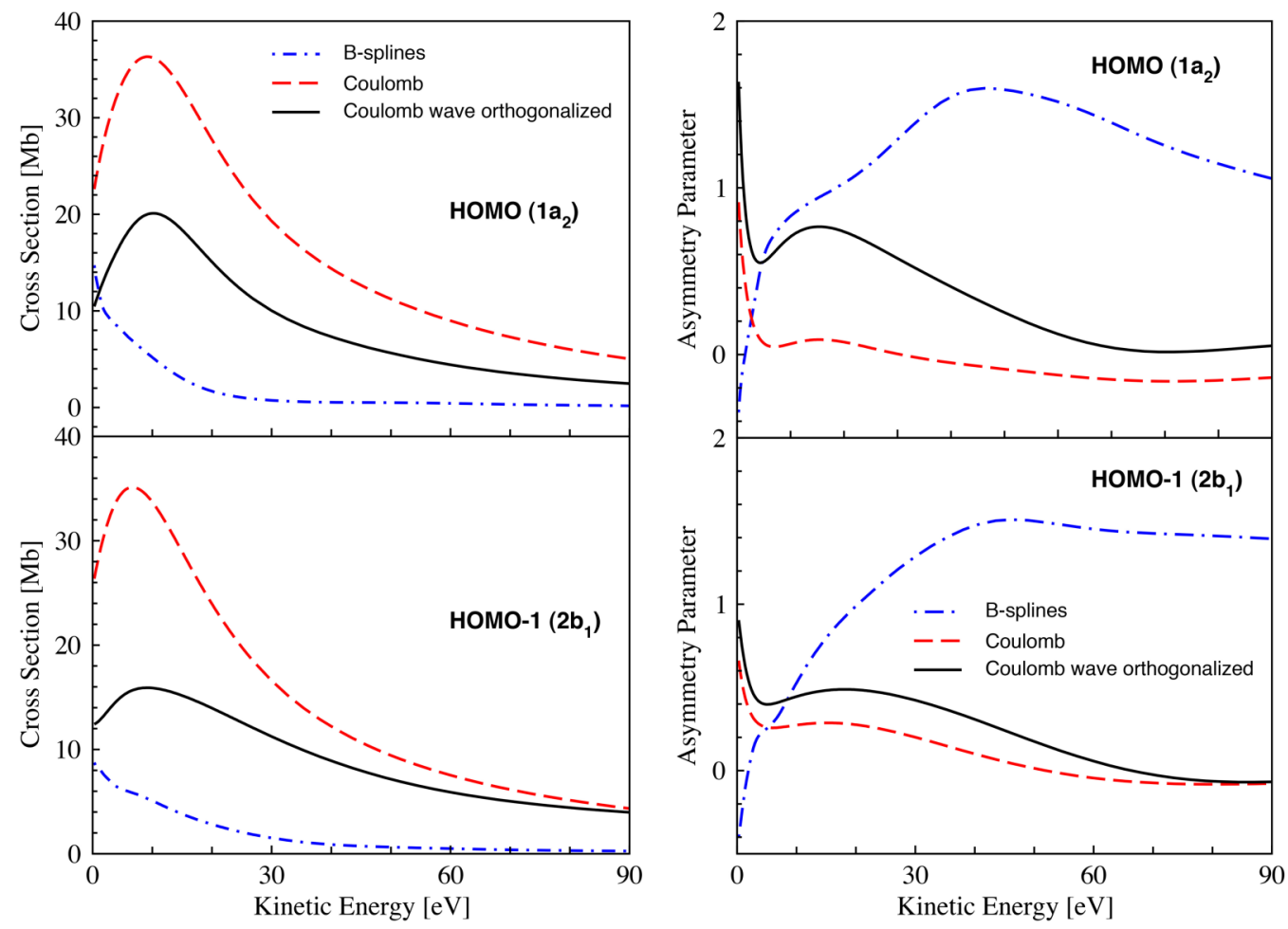

FIG. 6. The theoretical photoionization partial cross sections and photoelectron asymmetry parameters of the HOMO (upper panels) and HOMO-1 (lower panels) orbitals of furan, calculated with different treatments of the electronic continuum. 

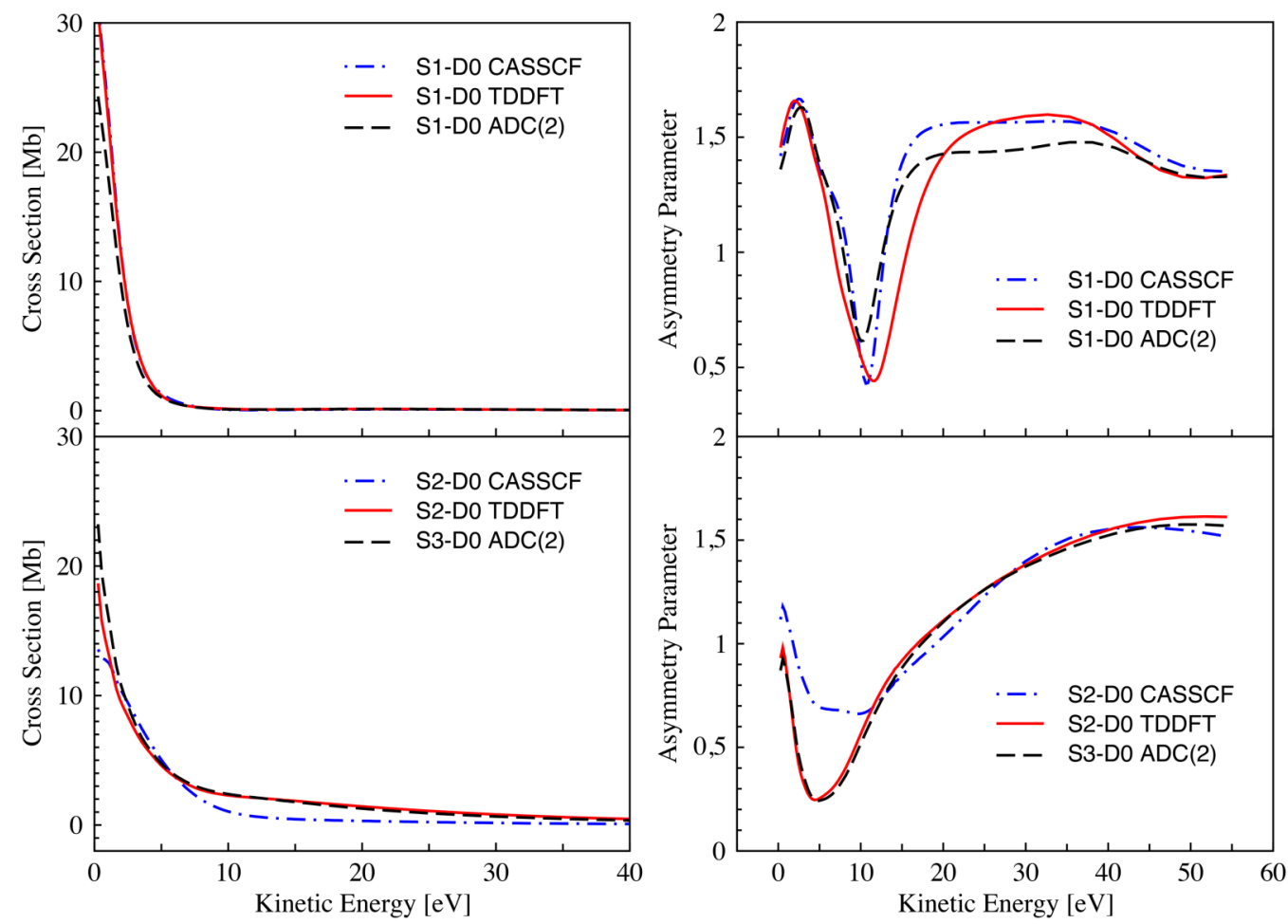

FIG. 7. Computed photoionization partial cross sections and photoelectron asymmetry parameters for the first transition (upper panels) and for the second transition (lower panels) of furan at equilibrium geometry.

conversion from the initially excited $\mathrm{S}_{2}$ to the ground state takes place within $\approx 50$ fs. ${ }^{11}$

We consider here the ionization from $S_{1}$ and $S_{2}$ states to the lowest state of the cation, $\mathrm{D}_{0}$. The Dyson orbital approach is used with the goal of investigating how the CASSCF, ADC(2), and TDDFT treatments of excited electronic states (bound states of the neutral) affect the photoionization observables. The CASSCF wave function of the lowest-lying singlet excited state $S_{1}$ contains one major configuration, corresponding to the transition from $\operatorname{HOMO}\left(1 \mathrm{a}_{2}\right)$ orbital to virtual orbital $10 \mathrm{a}_{1}$.
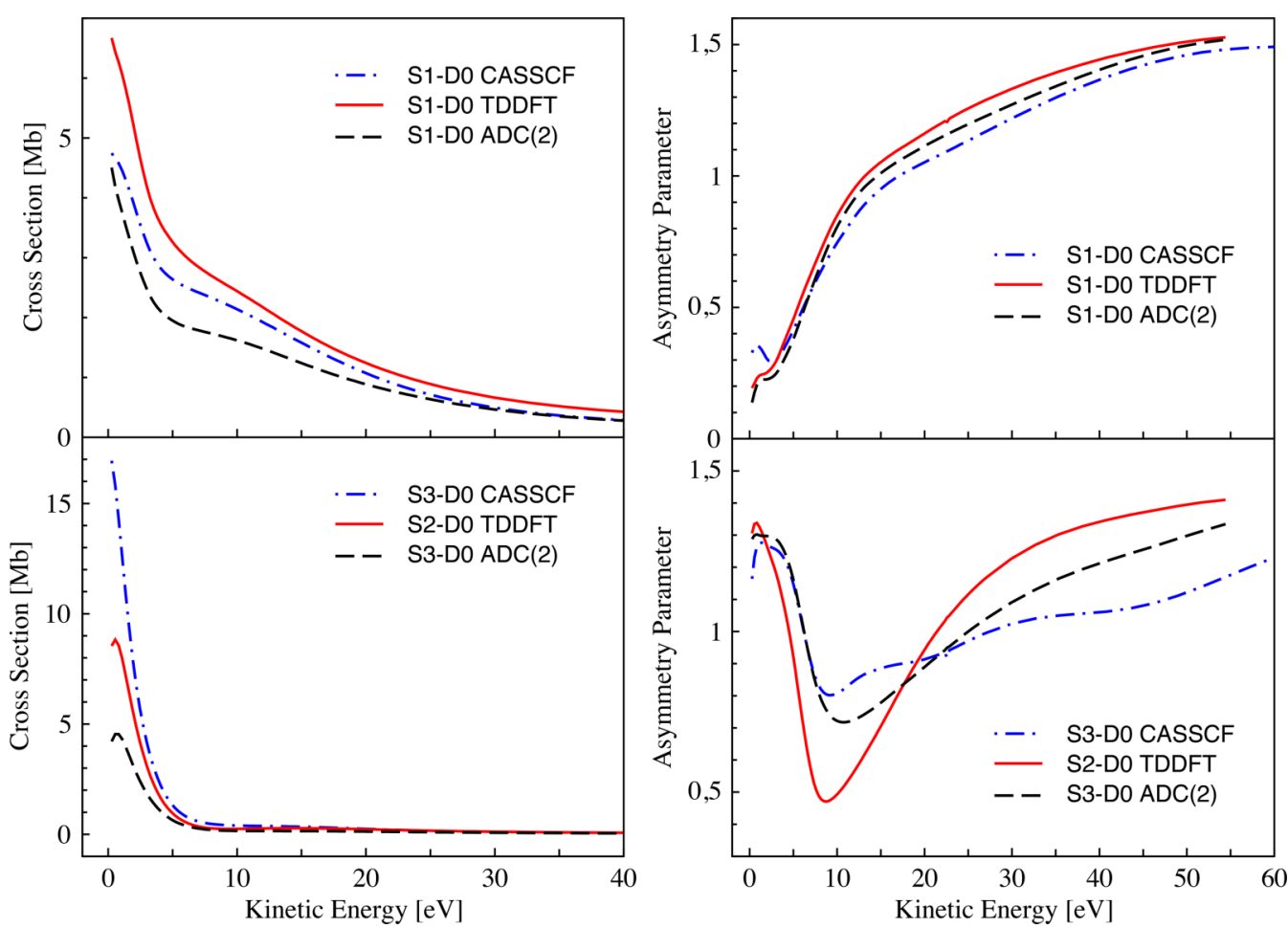

FIG. 8. The theoretical photoionization partial cross sections and photoelectron asymmetry parameters for the first transition (upper panels) and for the second transition (lower panels) of furan at a puckered geometry. 
The ground state of the cation derives from the one-electron ionization of this excited state. The corresponding Dyson orbital consists of the $10 \mathrm{a}_{1} \mathrm{MO}$ contribution (70\%), in addition to negligible contributions from other molecular orbitals of the same symmetry. The second excited state $S_{2}$ corresponds to excitation from the HOMO orbital to the $3 b_{1}$ virtual orbital. The Dyson orbital for this ${ }^{1} \mathrm{~B}_{2} \rightarrow{ }^{2} \mathrm{~A}_{2}$ transition is composed of the $3 b_{1} \mathrm{MO}(69 \%)$ with smaller contributions from the other $b_{1}$ molecular orbitals. The compositions of the Dyson orbitals reflect the weight of the larger CI coefficients in the dominant reference configuration of the initial $S_{1}$ and $S_{2}$ excited states: $68 \%$ for both cases. At the ADC(2) level, the Dyson orbital for the first transition is of $\mathrm{a}_{1}$ symmetry and is composed $79 \%$ from the $3 s \mathrm{MO}$. The $\mathrm{b}_{1}$ Dyson orbital corresponds to the third transition and it is composed of molecular orbitals $3 p_{x}(68 \%)$ and $\pi^{*}(17 \%)$. The TDDFT Dyson orbital for ionization from the $\mathrm{S}_{1}$ state is composed from the $3 s \mathrm{MO}(95 \%)$, while the one corresponding to ionization from the $S_{2}$ state is composed dominantly of the $\pi^{*}$ MO (76\%). The square norms of the Dyson orbitals corresponding to the two transitions of interest are collected in Table IV. As it can be seen, CASSCF, TDDFT, and $\mathrm{ADC}(2)$ provide similar results for the spectral strengths.

The computed cross sections and asymmetry parameters are shown in Figure 7. The cross sections are again relatively smooth and the only notable difference concerns the description of the ionization from the $S_{2}$ state where the CASSCF values decrease significantly faster with the energy increase. The asymmetry parameters, however, are much more sensitive to the character of the Dyson orbital. For the first transition, the $\beta$ profiles show modulations at intermediate energies in all three methods. The TDDFT profile presents a wider dip with a minimum shifted by about $2 \mathrm{eV}$ with respect

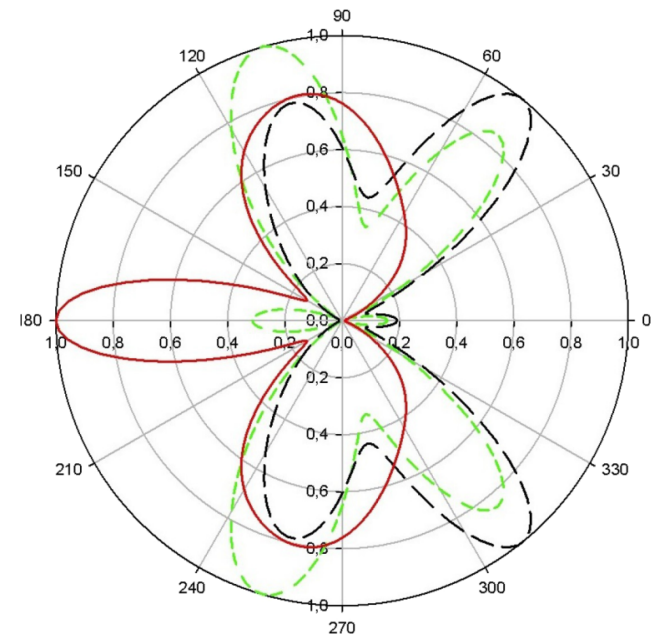

(a) $S_{1}-D_{0}, K E=5 \mathrm{eV}$

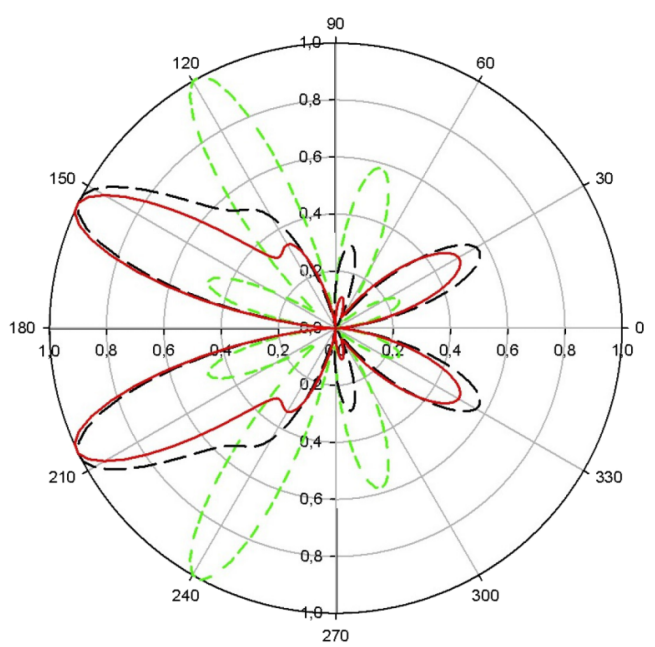

(c) $S_{2} / S_{3}-D_{0}, K E=5 e V$

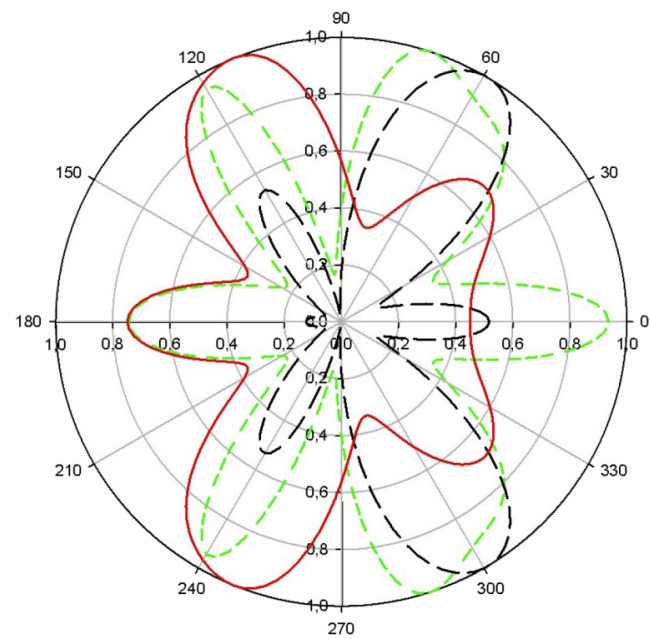

(b) $S_{1}-D_{0}, K E=10 \mathrm{eV}$

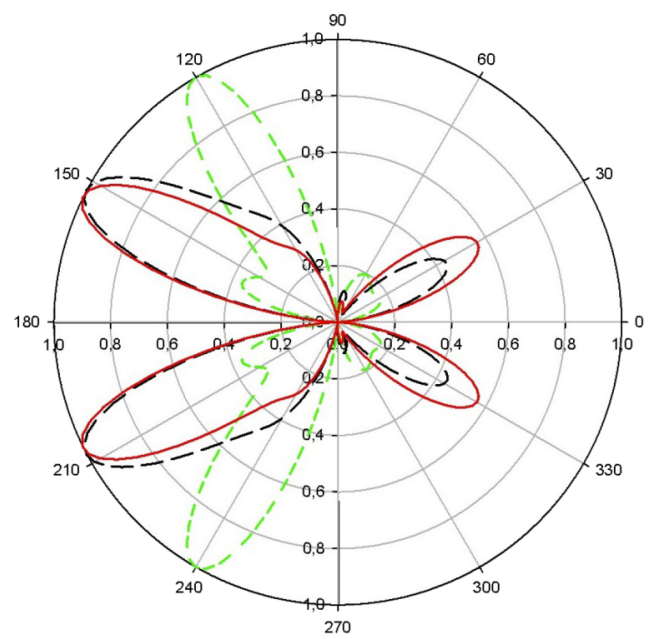

(d) $S_{2} / S_{3}-D_{0}, K E=10 \mathrm{eV}$

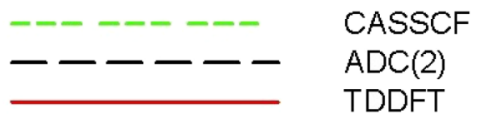

FIG. 9. Theoretical MFPADs for the first transition $\left(S_{1}-D_{0}\right.$, upper panel) and for the second transition $\left(S_{2} / S_{3}-D_{0}\right.$, lower panel) of furan at equilibrium geometry. Electron emission is in the molecular (YZ) plane. 
to the $\mathrm{ADC}(2)$ result. Further, the CASSCF results are higher than the $\mathrm{ADC}(2)$ ones by about $0.2 \beta$ units. In the case of the second transition, the TDDFT and ADC $(2)$ curves are basically superimposed having a minimum at $\sim 0.2 \beta$ units, whereas the one associated to the CASSCF results has a minimum value of $\sim 0.7 \beta$ units by around $5 \mathrm{eV}$ to higher energies. Owing to the different composition of TDDFT and ADC(2) Dyson orbitals in terms of MOs, the similarity of the two profiles was surprising. However, the very low square norm of the difference of the two Dyson orbitals computed in terms of Gaussian basis functions (0.014) proves that the orbitals are basically identical.

The sensitivity of the computed cross sections and $\beta$ parameters on the level of theory employed for the description of the excited states is even more pronounced in the bent geometry away from the Franck-Condon region, where the

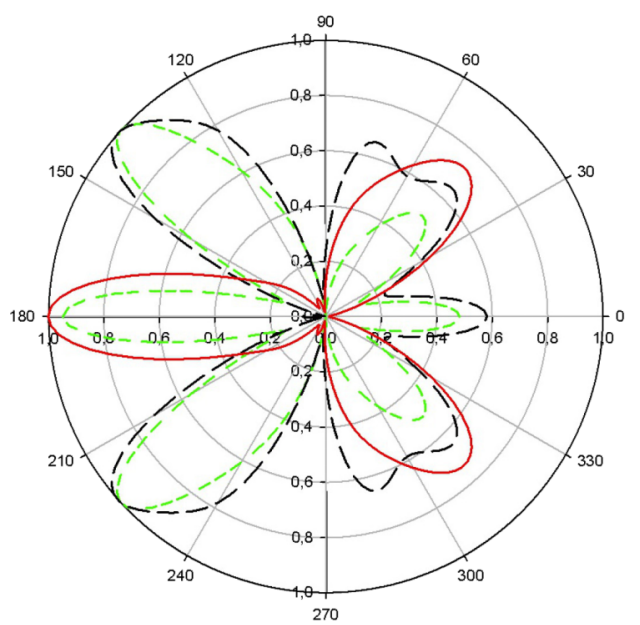

(a) $S_{1}-D_{0}, K E=5 e V$

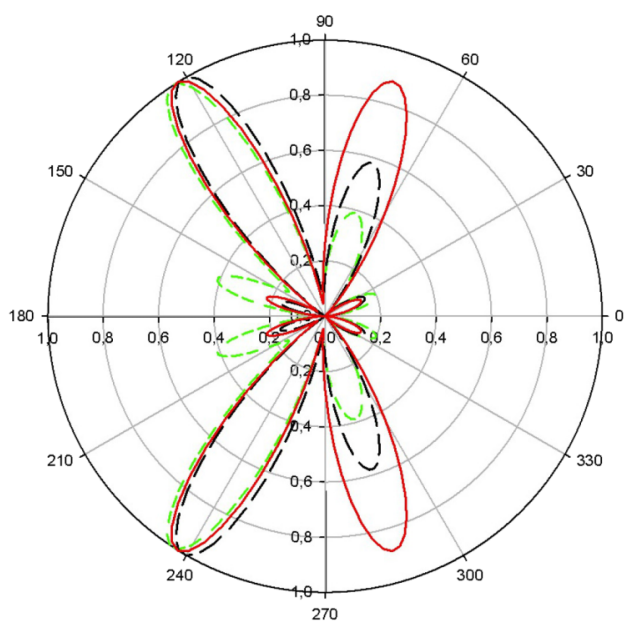

(c) $S_{2} / S_{3}-D_{0}, K E=5 \mathrm{eV}$ loss of symmetry allows further orbital mixing. Figure 8 displays the results for the puckered geometry of furan. Observables corresponding to states of equal character are compared. For the selected geometry, the TDDFT $\mathrm{S}_{2}$ state corresponds to the $\mathrm{S}_{3}$ state at the CASSCF and ADC(2) levels. TDDFT Dyson orbitals corresponding to the first and second transitions are of clear $\pi^{*}(89 \%)$ and $3 s(83 \%)$ characters. The corresponding ADC(2) Dyson orbitals consist of a combination of virtual orbitals. For ionization from $S_{1}$ and $\mathrm{S}_{3}$ states, the dominant contribution arises, respectively, from the $\pi^{*} \mathrm{MO}(54 \%)$, and from the $3 s$ MO with a contribution of only $42 \%$. The corresponding excitation energies and square norms of the Dyson orbitals are given in Tables III and IV. Here the norms are smaller and the differences among the methods larger, in particular, for the first transition at the $\mathrm{ADC}(2)$ level. Turning to the observables, it is immediately

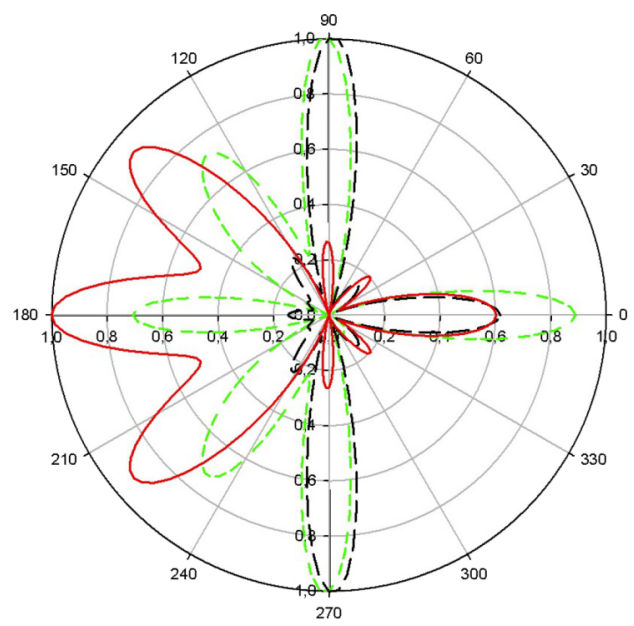

(b) $\mathrm{S}_{1}-\mathrm{D}_{0}, \mathrm{KE}=10 \mathrm{eV}$

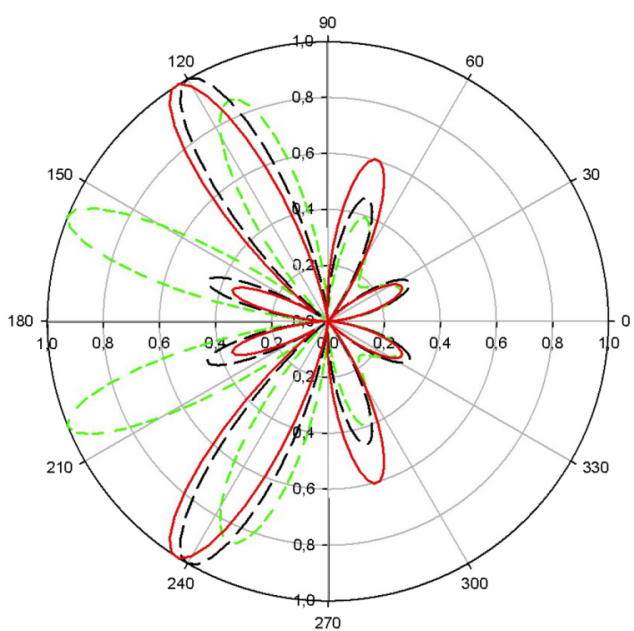

(d) $S_{2} / S_{3}-D_{0}, K E=10 \mathrm{eV}$

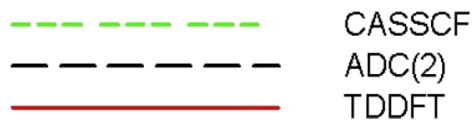

FIG. 10. Theoretical MFPADs for the first transition $\left(\mathrm{S}_{1}-\mathrm{D}_{0}\right.$, upper panel) and for the second transition $\left(\mathrm{S}_{2} / \mathrm{S}_{3}-\mathrm{D}_{0}\right.$, lower panel) of furan at equilibrium geometry. Electron emission is in the orthogonal (XZ) plane. 
evident that far from planarity, a more substantial difference exists between the three electronic structure methods, both in the cross sections and $\beta$ parameters. While the overall shape of the cross section curves is similar, the decay is less smooth than for the planar geometry and the ADC(2) values are consistently lower in agreement with the lower spectral strengths. The steeper decrease of the cross section in the case of the second transition is an indication of a more diffuse orbital, as derived from the well known inverse relationship between extension in space and momentum in Fourier transforms. As expected, significant differences are found in the asymmetry parameters for the second transition where the TDDFT curve has a minimum at $0.5 \beta$ units, whereas the CASSCF and ADC(2) profiles have minima at 0.7 and $0.8 \beta$ units, respectively. Also there is a noticeable difference in the high energy region.

In actual experiments, the excited molecules will be partially aligned by the excitation pulse, with a nonrandom statistical distribution. This can be considered by taking suitable averages of the molecular frame photoelectron angular distribution (MFPAD), as detailed, for instance, in Ref. 24, and will afford even more sensitive probe of the excited state electronic structure. In the absence of specific detail about molecular alignment, we have computed the full MFPAD, which represents the maximum information available before eventual averaging, for the two excited states of the planar molecule, at electron kinetic energies of $5 \mathrm{eV}$ and $10 \mathrm{eV}$. Actually MFPADs depend on the angles which specify both the polarization vector of the radiation and the direction of electron momentum. We have chosen for illustration two situations, with the electric vector along the $\mathrm{Z}$ axis and electron emission in the plane of the molecule (YZ plane) or in the orthogonal XZ plane. The relative results are reported in Figures 9 and 10, respectively. One immediately appreciates the extreme sensitivity of MFPADs to the details of the excited state description. For ionization out of $\mathrm{S}_{2} / \mathrm{S}_{3}$, TDDFT and $\mathrm{ADC}(2)$ appear rather similar, both in the $\mathrm{YZ}$ and $\mathrm{XZ}$ planes, while CASSCF shows significant differences, especially in the $\mathrm{YZ}$ plane. The situation is more complex for the $\mathrm{S}_{1}$ excited state. In the YZ plane, TDDFT deviates most from CASSCF, while $\mathrm{ADC}(2)$ is more similar to the latter, while in the $\mathrm{XZ}$ plane all three approaches are quite different, with CASSCF being very structured, like merging lobes found either in TDDFT or ADC(2). This underlies again the great sensitivity of fully angularly resolved photoemission, an ideal probe of the structural evolution of the system.

Altogether, the present results are quite encouraging, showing that photoionization observables obtained for the ionization of furan from excited electronic states are sensitive probes of the nature of the excited state and the quality of excited state wave functions. This opens the door for applications in more demanding situations such as TRPES. Furthermore, both TDDFT and ADC(2) methods provide, except for minor differences, Dyson orbitals comparable to those obtained by CASSCF, which can be used to describe TRPES observables along several non-adiabatic trajectories. However, an accurate treatment of the final continuum states is a necessary precondition for such applications since the differences among computational methods aimed at the description of bound states are masked by the errors introduced in the treatment of the continuum.

\section{CONCLUSIONS}

The goal of our study has been to perform a set of benchmark calculations for the photoionization of furan from the ground state and the two lowest-lying excited electronic states. We first performed calculations of the photoionization observables for the ground state of furan to assess the quality of the description of the continuum. Good agreement both with experimental branching ratios and angular distributions has been obtained. Next, bound states have been treated at the DFT and HF levels as well as with Dyson orbitals based on CASSCF calculation. Calculations have been performed for the cross sections and asymmetry parameters of HOMO and HOMO-1 orbitals to evaluate the difference in the treatment of ground state bound states. No significant difference has been found. On the contrary, when the Dyson orbitals approach has been used in combination with a more approximative treatment of the continuum, at the Coulomb and orthogonalized Coulomb levels, qualitatively different results have been obtained.

Furthermore, we have investigated the photoionization of furan from the first two excited-states to the first ionic state, both at the planar Franck-Condon geometry and at a puckered one. In this part, we have performed a comparative study of photoionization observables computed using the Dyson orbitals approach obtained by using three electronic structure methods: TDDFT, ADC(2), and CASSCF. The continuum has always been treated at the B-spline/DFT level. The difference among the three electronic structure methods was found to be more pronounced for the bent geometry than for the equilibrium geometry, both in the cross sections and $\beta$ parameters. The latter, in particular, have proven to be very sensitive to the character of the Dyson orbitals. This demonstrates that these dynamical observables are good probes of the quality of excited state wave functions. Also, despite minor differences, both TDDFT and ADC(2) provide Dyson orbitals that are quite adequate for the calculation of photoionization observables from the excited states. So, they can be used for an accurate interpretation of TRPES experiments. However, in addition to this, an accurate treatment of the continuum states is essential.

\section{ACKNOWLEDGMENTS}

The authors wish to thank D. M. P. Holland for providing us experimental data. This work has been supported by the Unity through Knowledge Fund (UKF B1) and the European COST Action CM1204 XLIC. Computer time was provided by CRONGI and CINECA. AP and MS acknowledge support of the European Union, European Social Fund under Call number HR.3.2.01 for the project Networks for professional training of young scientists in interdisciplinary research of innovative surfaces and materials (MIPoMaT).

${ }^{1}$ B. G. Levine and T. J. Martinez, Annu. Rev. Phys. Chem. 58, 613 (2007).

${ }^{2}$ Conical Intersections: Theory, Computation and Experiment, edited by W. Domcke, D. Yarkony, and H. Köppel (World Scientific, Singapore, 2011). 
${ }^{3}$ F. Plasser, M. Barbatti, A. Aquino, and H. Lischka, Theor. Chem. Acc. 131, 1073 (2012).

${ }^{4}$ B. F. E. Curchod, U. Rothlisberger, and I. Tavernelli, ChemPhysChem 14, 1314 (2013).

${ }^{5}$ T. Mori, W. J. Glover, M. S. Schuurman, and T. J. Martinez, J. Phys. Chem. A 116, 2808 (2011)

${ }^{6}$ M. Barbatti, Z. Lan, R. Crespo-Otero, J. J. Szymczak, H. Lischka, and W. Thiel, J. Chem. Phys. 137, 22A503 (2012).

${ }^{7}$ M. Sapunar, A. Ponzi, S. Chaiwongwattana, M. Mališ, A. Prlj, P. Decleva, and N. Došlić, Phys. Chem. Chem. Phys. 17, 19012 (2015).

${ }^{8}$ V. Stert, H. Lippert, H.-H. Ritze, and W. Radloff, Chem. Phys. Lett. 388, 144 (2004).

${ }^{9}$ A. Stolow, Annu. Rev. Phys. Chem. 54, 89 (2003).

${ }^{10}$ T. Suzuki, Annu. Rev. Phys. Chem. 57, 555 (2006)

${ }^{11}$ T. Suzuki, Int. Rev. Phys. Chem. 31, 265 (2012).

${ }^{12}$ S. Ullrich, T. Schultz, M. Z. Zgierski, and A. Stolow, J. Am. Chem. Soc. 126, 2262 (2004).

${ }^{13}$ H. Satzger, D. Townsend, M. Zgierski, S. Patchkovskii, S. Ullrich, and A. Stolow, Proc. Natl. Acad. Sci. U. S. A. 103, 10196 (2006).

${ }^{14}$ T. Horio, T. Fuji, Y.-I. Suzuki, and T. Suzuki, J. Am. Chem. Soc. 131, 10392 (2009).

${ }^{15}$ Y.-I. Suzuki, T. Fuji, T. Horio, and T. Suzuki, J. Chem. Phys. 132, 174302 (2010).

${ }^{16}$ T. Fuji, Y.-I. Suzuki, T. Horio, T. Suzuki, R. Mitrić, U. Werner, and V. Bonačić-Koutecký, J. Chem. Phys. 133, 234303 (2010).

${ }^{17}$ Y.-I. Suzuki, T. Horio, T. Fuji, and T. Suzuki, J. Chem. Phys. 134, 184313 (2011).

${ }^{18}$ T. Suzuki, J. Phys. B: At., Mol. Opt. Phys. 47, 124001-1 (2014).

${ }^{19}$ G. Wu, S. P. Neville, O. Schalk, T. Sekikawa, M. N. R. Ashfold, G. A. Worth, and A. Stolow, J. Chem. Phys. 142, 074302 (2015).

${ }^{20} \mathrm{G}$. Wu, P. Hockett, and A. Stolow, Phys. Chem. Chem. Phys. 13, 18447 (2011).

${ }^{21}$ A. Ponzi, C. Angeli, R. Cimiraglia, S. Coriani, and P. Decleva, J. Chem. Phys. 140, 204304 (2014).

${ }^{22}$ H. Bachau, E. Cormier, P. Decleva, J. E. Hansen, and F. Martin, Rep. Prog. Phys. 64, 1815 (2001).

${ }^{23}$ H. Tao, T. K. Allison, T. W. Wright, A. M. Stooke, C. Khurmi, J. van Tilborg, Y. Liu, R. W. Falcone, A. Belkacem, and T. J. Martinez, J. Chem. Phys. 134, 244306 (2011)

${ }^{24}$ A. Humeniuk, M. Wohlgemuth, T. Suzuki, and R. Mitrić, J. Chem. Phys. 139, 134104 (2013).

${ }^{25}$ G. Tomasello, A. Humeniuk, and R. Mitrić, J. Phys. Chem. A 118, 8437 (2014).

${ }^{26}$ C. M. Oana and A. I. Krylov, J. Chem. Phys. 127, 234106 (2007).

${ }^{27}$ M. Walter and H. Häkkinen, New J. Phys. 10, 043018 (2008).

${ }^{28}$ C. M. Oana and A. I. Krylov, J. Chem. Phys. 131, 124114 (2009).
${ }^{29}$ U. Werner, R. Mitrić, and V. Bonačić-Koutecký, J. Chem. Phys. 132, 174301 (2010).

${ }^{30}$ D. M. P. Holland, L. Karlsson, and W. von Niessen, J. Electron Spectrosc. Relat. Phenom. 113, 221 (2001).

${ }^{31}$ Y.-I. Suzuki and T. Suzuki, J. Phys. Chem. A 112, 402 (2008).

${ }^{32}$ D. Toffoli, M. Stener, G. Fronzoni, and P. Decleva, Chem. Phys. 276, 25 (2002).

${ }^{33}$ H. J. Werner, P. J. Knowles, G. Knizia, F. R. Manby, and M. Schütz, MOLPRo, version 2012.1, a package of ab initio programs, 2012, see http://www. molpro.net.

${ }^{34}$ TURBOMOLE V6.4 2012, a development of University of Karlsruhe and Forschungszentrum Karlsruhe GmbH, 1989-2007, TURBOMOLE GmbH, since 2007, available from http://www.turbomole.com.

${ }^{35}$ M. Stener, G. Fronzoni, and P. Decleva, J. Chem. Phys. 122, 234301 (2005).

${ }^{36}$ M. E. Casida, in Recent Developments and Applications in Modern DensityFunctional Theory, edited by J. M. Seminario (Elsevier, Singapore, 1996).

${ }^{37}$ E. Tapavicza, I. Tavernelli, and U. Rothlisberger, Phys. Rev. Lett. 98, 023001-1 (2007).

${ }^{38}$ T. X. Carroll, M. G. Zahl, K. J. Børve, L. J. Sæthre, P. Decleva, A. Ponzi, J. J. Kas, F. D. Vila, J. J. Rehr, and T. Darrah Thomas, J. Chem. Phys. 138, 234310 (2013).

${ }^{39}$ R. K. Kushawaha, M. Patanen, R. Guillemin, L. Journel, C. Miron, M. Simon, M. N. Piancastelli, and P. Decleva, Proc. Natl. Acad. Sci. U. S. A. 110, 15201 (2013).

${ }^{40}$ G. te Velde, F. M. Bickelhaupt, E. J. Baerends, C. F. Guerra, S. J. A. van Gisbergen, J. Snijders, and T. Ziegler, J. Comput. Chem. 22, 931 (2001).

${ }^{41}$ ADF2014, SCM, Theoretical Chemistry, Vrije Universiteit, Amsterdam, The Netherlands, http://www.scm.com.

${ }^{42}$ C. F. Fischer and M. Idrees, Comput. Phys. 3, 53 (1989).

${ }^{43}$ M. Brosolo and P. Decleva, Chem. Phys. 159, 185 (1992).

${ }^{44}$ M. Brosolo, P. Decleva, and A. Lisini, Comput. Phys. Commun. 71, 207 (1992).

${ }^{45}$ N. Chandra, J. Phys. B: At. Mol. Phys. 20, 3405 (1987).

${ }^{46}$ R. van Leeuwen and E. Baerends, Phys. Rev. A 49, 2421 (1994).

${ }^{47}$ T. H. Dunning, J. Chem. Phys. 90, 1007 (1989).

${ }^{48}$ C. Angeli, R. Cimiraglia, S. Evangelisti, T. Leininger, and J. P. Malrieu, J. Chem. Phys. 114, 10252 (2001).

${ }^{49}$ C. Angeli, R. Cimiraglia, and J. P. Malrieu, J. Chem. Phys. 117, 9138 (2002).

${ }^{50} \mathrm{~K}$. Kimura, S. Katsumata, Y. Achiba, T. Yamazaki, and S. Iwata, Handbook of He I Photoelectron Spectra of Fundamental Organic Molecules (Japan Scientific, Tokyo, 1981).

${ }^{51}$ P. R. Keller, J. W. Taylor, T. A. Carlson, and F. A. Grimm, J. Electron Spectrosc. Relat. Phenom. 33, 333 (1984).

${ }^{52}$ M. Pastore, C. Angeli, and R. Cimiraglia, Chem. Phys. Lett. 426, 445 (2006).

${ }^{53}$ F. Mata, M. C. Martin, and G. O. Sørensen, J. Mol. Struct. 48, 157 (1978). 